\title{
CORRIGENDUM
}

\section{Analysis of microsatellite markers at the UCP2/UCP3 locus on chromosome 11q13 in anorexia nervosa}

X Hu, F Murphy, A Karwautz, T Li, O Giotakis, J Treasure, DA Collier. Mol Psychiatry (2002) 7: 276-277. doi:10.1038/sj.mp.4001044

Molecular Psychiatry (2002) 7, 814. doi:10.1038/sj.mp.4001156

The authors wish to apologise that the labelling of the allele peaks in Figure 1 on page 277 is incorrect. The sizes shown are the sizes automatically assigned by the Applied Biosystems Genotyper program (156, 174, 180,
182, 190, 198 base pairs). In fact the true allele sizes, determined by sequencing PCR products are all three base pairs greater, and should read 159, 177, 183, 185, 193 and 201 base pairs. 